\title{
Birge and Qi Method for Three-Stage Stochastic Programs Using IPM
}

\author{
Georg Ch. Pflug and Ladislav Halada \\ Department of Statistics and Decision Support Systems, Vienna University, \\ Universitaesstr. 5, A-1010 Wien, Austria, \\ e-mail:georg.pflug@univie.ac.at
}

\begin{abstract}
One approach how to solve a linear optimization is based on interior point method. This method requires the solution of large linear system equations. A special matrix factorization techniques that exploit the structure of the constraint matrix has been suggested for its computation. The method of Birge and Qi has been reported as efficient, stable and accurate for two-stage stochastic programs. In this report we present a generalization of this method for three-stage stochastic programs.
\end{abstract}

\section{Introduction}

Solving the deterministic equivalent formulation of two-stage stochastic programs using interior point method requires the solution of linear systems of the form

$$
\left(A D A^{t}\right) d y=b .
$$

Solving of this problem requires more then $90-95 \%$ of total programming time [1]. Birge and Holmes [2] compared different methods for the solution of this system. They found that the factorization technique based on the work of Birge and Qi (BQ) [3] is more efficient and stable than other methods. They also suggested BQ for parallel computation. A parallel version of BQ for two-stage stochastic programs was also implemented on an Intel iPSC/860 hypercube and a Connection Machine CM-5 with nearly perfect speedup [4]. According to our knowledge, this method has not been used for a three-stage stochastic program so far. The aim of this report is analysis of the BQ method and a suggestion how the BQ method can be used for three-stage stochastic programming. Sect. 2 briefly describes the BQ matrix decomposition. An application of the BQ to a three-stage stochastic model is given in Sect. 3 .

\section{BQ Factorization for Two-Stage Model}

For expositional clarity, we start with a two-stage model which has been resolved in [2]. Let $A^{(2)}$ be a constraint matrix of the two-stage stochastic model and $D^{(2)}$ 
a positive definite and diagonal matrix

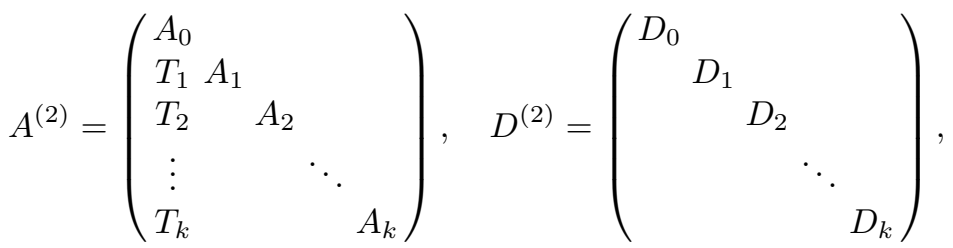

where $A_{i}$ are $m_{i} \times n_{i}$ and $D_{i}$ are positive definite diagonal $n_{i} \times n_{i}$ matrices, $i=0,1,2, \ldots, k . T_{i}$ are $m_{i} \times n_{0}$ matrices, $i=1,2, \ldots, k$. We assume that $A_{i}$ have full row rank and $m_{i} \leq n_{i}, i=0,1,2, \ldots, k$. For wit sake of completness, let us denote $R^{(2)}=A^{(2)} D^{(2)}\left(A^{(2)}\right)^{t}$. Then $R^{(2)}$ can be expressed as sum of a block-diagonal matrix and product of matrices

$$
R^{(2)}=\mathcal{R}^{(2)}+U^{(2)}\left[\mathcal{D}^{(2)}\left(V^{(2)}\right)^{t}\right]=\mathcal{R}^{(2)}+U^{(2)}\left[\left(W^{(2)}\right)^{t}\right]
$$

where

$$
\mathcal{R}^{(2)}=\operatorname{Diag}\left(I_{m_{0}}, A_{1} D_{1} A_{1}^{t}, \ldots, A_{k} D_{k} A_{k}^{t}\right)=\operatorname{Diag}\left(I_{m_{0}}, R_{1}, \ldots, R_{k}\right)
$$

and

$$
U^{(2)} \mathcal{D}^{(2)}\left(V^{(2)}\right)^{t}=\left(\begin{array}{c}
A_{0} \\
I_{m_{0}} \\
T_{1} \\
T_{2} \\
\vdots \\
T_{k}
\end{array}\right)\left(\begin{array}{cc}
D_{0} & \\
& I_{m_{0}}
\end{array}\right)\left(\begin{array}{cc}
A_{0}^{t} & T_{1}^{t} T_{2}^{t} \ldots T_{k}^{t} \\
-I_{m_{0}} &
\end{array}\right)
$$

Note $\mathcal{R}^{(2)}$ is the diagonal matrix with positive definite matrices on the diagonal entries. Now, if we need the inverse of $R^{(2)}$, we can use the Sherman-MorrisonWoodbury formula. It holds [5]

$$
\left(R^{(2)}\right)^{-1}=\left(\mathcal{R}^{(2)}\right)^{-1}-\left(\mathcal{R}^{(2)}\right)^{-1} U^{(2)}\left(G^{(2)}\right)^{-1}\left(V^{(2)}\right)^{t}\left(\mathcal{R}^{(2)}\right)^{-1},
$$

if and only if both $\mathcal{R}^{(2)}$ and $G^{(2)}$ are nonsingular, where

$$
\left(G^{(2)}\right)^{-1}=\left[I_{n_{0}+m_{0}}+\left(W^{(2)}\right)^{t}\left(\mathcal{R}^{(2)}\right)^{-1} U^{(2)}\right]^{-1} \mathcal{D}^{(2)} .
$$

In the matrix form

$$
G^{(2)}=\left(\begin{array}{cc}
D_{0}^{-1}+A_{0}^{t} A_{0}+\sum_{i=1}^{k} T_{i}^{t} R_{i}^{-1} T_{i} & A_{0}^{t} \\
-A_{0} & 0
\end{array}\right)=\left(\begin{array}{cc}
\hat{G}^{(2)} & A_{0}^{t} \\
-A_{0} & 0
\end{array}\right) .
$$

It has been proved [2] that $\hat{G}^{(2)}$ is positive definite and symmetric matrix and $G^{(2)}$ is nonsingular. Hence, the conditions for the validity of (6) are fulfilled. 
Thus, we can rewrite the solution of the system $R^{(2)} d y^{(2)}=b$ by the relation (6) as follows: $d y^{(2)}=p^{(2)}-s^{(2)}$, where

$$
\begin{aligned}
& \mathcal{R}^{(2)} p^{(2)}=b \\
& G^{(2)} q^{(2)}=\left(V^{(2)}\right)^{t} p^{(2)} \\
& \mathcal{R}^{(2)} s^{(2)}=U^{(2)} q^{(2)} .
\end{aligned}
$$

More precisely,

$$
\mathcal{R}^{(2)} p^{(2)}=\left(\begin{array}{ccccc}
I_{m_{0}} & & & \\
& R_{1} & & \\
& & \ddots & \\
& & & R_{k}
\end{array}\right)\left(\begin{array}{c}
p_{0}^{(2)} \\
p_{1}^{(2)} \\
\vdots \\
p_{k}^{(2)}
\end{array}\right)=\left(\begin{array}{c}
b_{0} \\
b_{1} \\
\vdots \\
b_{k}
\end{array}\right) .
$$

Hence, the vector $p^{(2)}$ can be computed component-wise by solving sub-block systems

$$
\begin{aligned}
p_{0}^{(2)} & =b_{0} \\
R_{i} p_{i}^{(2)} & =b_{i}, \quad i=1,2, \ldots k .
\end{aligned}
$$

We can proceed similarly in the case of computation of the vector $s^{(2)}$. It gives

$$
\begin{aligned}
s_{0}^{(2)} & =A_{0} q_{1}^{(2)}+q_{2}^{(2)} \\
R_{i} s_{i}^{(2)} & =T_{i} q_{1}^{(2)}, \quad i=1,2, \ldots k .
\end{aligned}
$$

Because $R_{i}=A_{i} D_{i} A_{i}^{t}, \quad i=1,2, \ldots k$ is symmetric positive definite matrix, its Cholesky decomposition can be used for the solution of both (13) and (14). The solution of (10) can be found by exploiting the matrix structure $G^{(2)}$. We have

$$
G^{(2)} q^{(2)}=\left(\begin{array}{cc}
\hat{G}^{(2)} & A_{0}^{t} \\
-A_{0} & 0
\end{array}\right)\left(\begin{array}{c}
q_{1}^{(2)} \\
q_{2}^{(2)}
\end{array}\right)=\left(\begin{array}{c}
\hat{v}_{1}^{(2)} \\
\hat{v}_{2}^{(2)}
\end{array}\right),
$$

where

$$
\left(\begin{array}{c}
\hat{v}_{1}^{(2)} \\
\hat{v}_{2}^{(2)}
\end{array}\right)=\left(V^{(2)}\right)^{t} p^{(2)}=\left(\begin{array}{c}
A_{0}^{t} p_{0}^{(2)}+\sum_{i=1}^{k} T_{i}^{t} p_{i}^{(2)} \\
-p_{0}^{(2)}
\end{array}\right) .
$$

The vectors $q_{i}^{(2)}$ and $\hat{v}_{i}^{(2)}, \mathrm{i}=1,2$ have the size corresponding to the matrix structure $G^{(2)}$. By an elimination process, for (15) we get

$$
\begin{aligned}
{\left[A_{0}\left(\hat{G}^{(2)}\right)^{-1} A_{0}^{t}\right] q_{2}^{(2)} } & =A_{0}\left(\hat{G}^{(2)}\right)^{-1} \hat{v}_{1}^{(2)}+\hat{v}_{2}^{(2)} \\
\hat{G}^{(2)} q_{1}^{(2)} & =\hat{v}_{1}^{(2)}-A_{0}^{t} q_{2}^{(2)} .
\end{aligned}
$$

Because both matrices $A_{0}\left(\hat{G}^{(2)}\right)^{-1} A_{0}^{t}$ and $\hat{G}^{(2)}$ are symmetric positive definite, one can use their Cholesky decomposition for solving these systems. The procedure for sequential computing of the vector $d y^{(2)}$ by (9)-(11) has been named 
Findy in [2]. The parallel version of Findy has been formulated in [4]. Formally, parameters of this procedure are $\operatorname{Findy}\left(\mathcal{R}^{(2)}, A_{0}, D_{0}, T_{1}, \ldots, T_{k}, b, d y^{(2)}\right)$. In [4] this procedure has been implemented on a distributed-memory multipleinstruction multiple-date (MIMD) message-passing parallel computers, an Intel iPSC/860 hypercube and a Connection Machine CM-5. Results are reported with the solution of the linear systems arising when solving stochastic programs with 98, 304 scenarios, which correspond to deterministic equivalent linear programs with up to 1,966, 090 constraints and 13,762,630 variables. From the timing data presented in this paper it is evident that the speed-up and the efficiency is most influenced by the percentage of time spent in communication and also by the ratio $m_{i} / n_{i}$.

\section{BQ Method Applied to Three-Stage Stochastic Model}

Let $A^{(3)}$ be here a constraint matrix of a three-stage stochastic model

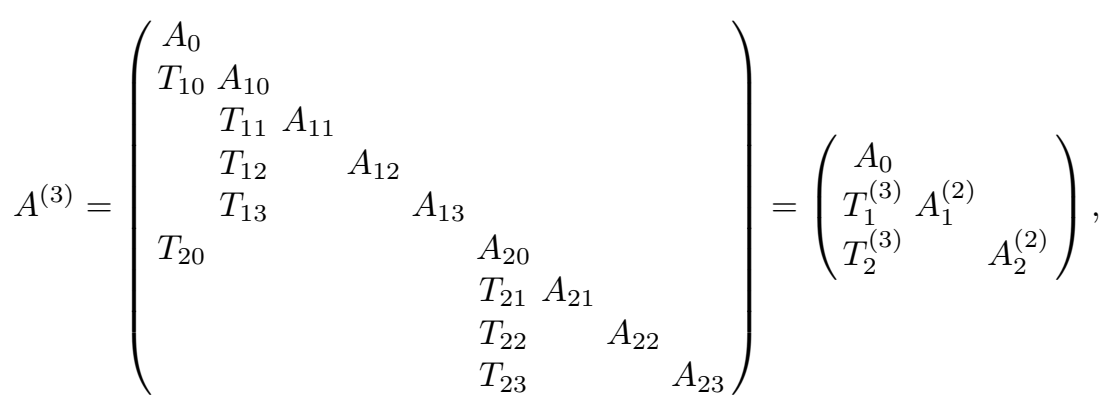

where $A_{0}$ is $m_{0} \times n_{0}$ and $A_{i j}$ are $m_{i j} \times n_{i j}$ matrices. $T_{i j}$ has the size conformable to the matrices $A_{0}$ and $A_{i j}$. Let $D^{(3)}$ be diagonal and positive definite matrix

$$
D^{(3)}=\operatorname{Diag}\left(D_{0} ; D_{10}, \ldots, D_{13} ; D_{20}, \ldots, D_{23}\right)=\operatorname{Diag}\left(D_{0}, D_{1}^{(2)}, D_{2}^{(2)}\right),
$$

where $D_{0}$ and $D_{i j}$ are diagonal $n_{0} \times n_{0}$ and $n_{i j} \times n_{i j}$ matrices with positive entries, $i=1,2 ; j=0,1,2,3$. Again, we assume that $A_{0}$ and $A_{i j}$ have full row rank and $m_{0} \leq n_{0}, \quad m_{i j} \leq n_{i j}, \quad i=1,2 ; j=0,1,2,3$.

The matrix $R^{(3)}=A^{(3)} D^{(3)}\left(A^{(3)}\right)^{t}$ in the denotation of the largesized blocks matrix $A^{(3)}$ can be decomposed as follows:

$$
R^{(3)}=\mathcal{R}^{(3)}+U^{(3)}\left[\mathcal{D}^{(3)}\left(V^{(3)}\right)^{t}\right]=\mathcal{R}^{(3)}+U^{(3)}\left[\left(W^{(3)}\right)^{t}\right]
$$

where

$$
\mathcal{R}^{(3)}=\operatorname{Diag}\left(I_{m_{0}}, A_{1}^{(2)} D_{1}^{(2)}\left(A_{1}^{(2)}\right)^{t}, A_{2}^{(2)} D_{2}^{(2)}\left(A_{2}^{(2)}\right)^{t}\right)=\operatorname{Diag}\left(I_{m_{0}}, R_{1}^{(2)}, R_{2}^{(2)}\right)
$$


and

$$
U^{(3)} \mathcal{D}^{(3)}\left(V^{(3)}\right)^{t}=\left(\begin{array}{ll}
A_{0} & I_{m_{0}} \\
T_{1}^{(3)} & \\
T_{2}^{(3)} &
\end{array}\right)\left(\begin{array}{cc}
D_{0} & \\
& I_{m_{0}}
\end{array}\right)\left(\begin{array}{cc}
A_{0}^{t} & \left(T_{1}^{(3)}\right)^{t}\left(T_{2}^{(3)}\right)^{t} \\
-I_{m_{0}} &
\end{array}\right)
$$

By the Sherman-Morrison-Woodbury formula we obtain again

$$
\left(R^{(3)}\right)^{-1}=\left(\mathcal{R}^{(3)}\right)^{-1}-\left(\mathcal{R}^{(3)}\right)^{-1} U^{(3)}\left(G^{(3)}\right)^{-1}\left(V^{(3)}\right)^{t}\left(\mathcal{R}^{(3)}\right)^{-1},
$$

if and only if $\mathcal{R}^{(3)}$ and $G^{(3)}$ are nonsingular. Here

$$
\begin{aligned}
\left(G^{(3)}\right)^{-1} & =\left[I_{n_{0}+m_{0}}+\mathcal{D}^{(3)}\left(V^{(3)}\right)^{t}\left(\mathcal{R}^{(3)}\right)^{-1} U^{(3)}\right]^{-1} \mathcal{D}^{(3)} \\
G^{(3)} & =\left(\mathcal{D}^{(3)}\right)^{-1}+\left(V^{(3)}\right)^{t}\left(\mathcal{R}^{(3)}\right)^{-1} U^{(3)}
\end{aligned}
$$

In the matrix form:

$$
G^{(3)}=\left(\begin{array}{cc}
D_{0}^{-1}+A_{0}^{t} A_{0}+\sum_{i=1}^{2}\left(T_{i}^{(3)}\right)^{t}\left(R_{i}^{(2)}\right)^{-1} T_{i}^{(3)} & A_{0}^{t} \\
-A_{0} & 0
\end{array}\right)=\left(\begin{array}{cc}
\hat{G}^{(3)} & A_{0}^{t} \\
-A_{0} & 0
\end{array}\right) .
$$

The validity of (23) follows from the same reason as for the two-stage model. Thus, the solution of $R^{(3)} d y^{(3)}=b^{(3)}$ can be expressed by the inversion as $d y^{(3)}=p^{(3)}-s^{(3)}$ while

$$
\begin{aligned}
& \mathcal{R}^{(3)} p^{(3)}=b^{(3)} \\
& G^{(3)} q^{(3)}=\left(V^{(3)}\right)^{t} p^{(3)} \\
& \mathcal{R}^{(3)} s^{(3)}=U^{(3)} q^{(3)}
\end{aligned}
$$

The equations (26)-(28) represent the decomposition of the original problem into three sub-problems. An advantage of such decomposition is that $\mathcal{R}^{(3)}$ is the block-diagonal matrix amenable to further decomposition.

\subsection{Solving the Equation $\mathcal{R}^{(3)} p^{(3)}=b^{(3)}$}

It is easy to see from the equation

$$
\mathcal{R}^{(3)} p^{(3)}=\left(\begin{array}{ccc}
I_{m_{0}} & & \\
& R_{1}^{(2)} & \\
& & R_{2}^{(2)}
\end{array}\right)\left(\begin{array}{c}
p_{0}^{(3)} \\
p_{1}^{(3)} \\
p_{2}^{(3)}
\end{array}\right)=\left(\begin{array}{c}
b_{0}^{(3)} \\
b_{1}^{(3)} \\
b_{2}^{(3)}
\end{array}\right)
$$

that this system represents the following independent systems

$$
\begin{aligned}
p_{0}^{(3)} & =b_{0}^{(3)} \\
R_{i}^{(2)} p_{i}^{(3)} & =b_{i}^{(3)}, \quad i=1,2,
\end{aligned}
$$


where $R_{i}^{(2)}=A_{i}^{(2)} D_{i}^{(2)}\left(A_{i}^{(2)}\right)^{t}, i=1,2$ represent the matrix of the two-stage model problem, which has been described in Sect.2. Therefore, (31) can be solved by the procedure Findy or Parallel Findy, depending on the number of processors. Its input parameters are readable from the entries of matrix $A_{i}^{(2)}, D_{i}^{(2)}$ defined in (18)-(19). The right-hand side and the solution vector are $b_{i}^{(3)}$ and $p_{i}^{(3)}, i=1,2$, respectively. It is clear that in our case the parameters are

$$
\operatorname{Findy}\left(\mathcal{R}_{i}^{(2)}, A_{i 0}, D_{i 0}, T_{i 1}, \ldots, T_{i 3}, b_{i}^{(3)}, p_{i}^{(3)}\right), \quad i=1,2
$$

where $\mathcal{R}_{i}^{(2)}$ is the diagonal matrix in the decomposition $R_{i}^{(2)}$, i.e.

$$
R_{i}^{(2)}=\mathcal{R}_{i}^{(2)}+U_{i}^{(2)}\left(W_{i}^{(2)}\right)^{t} \quad i=1,2
$$

and

$$
\begin{aligned}
& \mathcal{R}_{i}^{(2)}=\operatorname{Diag}\left(I_{m_{i 0}}, R_{i 1}, R_{i 2}, R_{i 3}\right), \quad R_{i j}=A_{i j} D_{i j} A_{i j}^{t}, \quad i=1,2 \quad j=1,2,3
\end{aligned}
$$

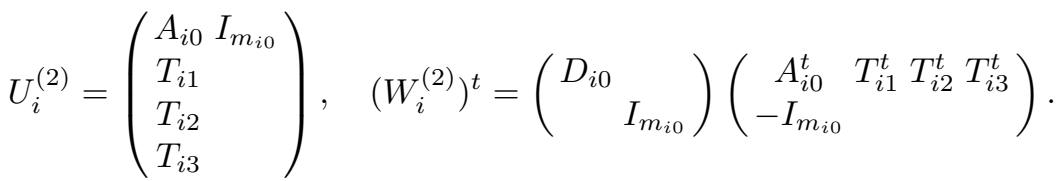

If this procedure is applied for the given values, having in the mind the relations (30)-(31) we are able to compose the vector $p^{(3)}$.

\subsection{Solving the Equation $G^{(3)} q^{(3)}=\left(V^{(3)}\right)^{t} p^{(3)}$}

Solving of this equation requires to have the entries of the right-hand side vector and the sub-block matrix $\hat{G}^{(3)}$ available. With this aim we denote the elements of the vector $\left(V^{(3)}\right)^{t} p^{(3)}$ as $\left(\hat{v}_{1}^{(3)}, \hat{v}_{2}^{(3)}\right)^{t}$. Then we have

$$
\left(\begin{array}{c}
\hat{v}_{1}^{(3)} \\
\hat{v}_{2}^{(3)}
\end{array}\right)=\left(\begin{array}{c}
A_{0}^{t} p_{0}^{(3)}+\sum_{i=1}^{2} T_{i 0}^{t} p_{i 0}^{(3)} \\
-p_{0}^{(3)}
\end{array}\right),
$$

where $p_{i 0}^{(3)}, i=1,2$ is the vector of the first $m_{i 0}$-elements of $p_{i}^{(3)}$. We know from (25) that

$$
\hat{G}^{(3)}=D_{0}^{-1}+A_{0}^{t} A_{0}+\sum_{i=1}^{2}\left(T_{i}^{(3)}\right)^{t}\left(R_{i}^{(2)}\right)^{-1} T_{i}^{(3)} .
$$

For the relatively complicated expression $\left(T_{i}^{(3)}\right)^{t}\left(R_{i}^{(2)}\right)^{-1} T_{i}^{(3)}$ we can prove that

$$
\left(T_{i}^{(3)}\right)^{t}\left(R_{i}^{(2)}\right)^{-1} T_{i}^{(3)}=T_{i 0}^{t}\left(\hat{T}_{i 0}-T_{i 0}\right), \quad i=1,2
$$

where $\hat{T}_{i 0}$ is the solution of the equation

$$
\left[A_{i 0}\left(\hat{G}_{i}^{(2)}\right)^{(-1)} A_{i 0}^{t}\right] \hat{T}_{i 0}=T_{i 0}, \quad i=1,2 .
$$


Really, according to (6) we have

$$
\begin{gathered}
\left(T_{i}^{(3)}\right)^{t}\left(R_{i}^{(2)}\right)^{-1} T_{i}^{(3)}= \\
\left(T_{i}^{(3)}\right)^{t}\left[\left(\mathcal{R}_{i}^{(2)}\right)^{-1}-\left(\mathcal{R}_{i}^{(2)}\right)^{-1} U_{i}^{(2)}\left(G_{i}^{(2)}\right)^{-1}\left(V_{i}^{(2)}\right)^{t}\left(\mathcal{R}_{i}^{(2)}\right)^{-1}\right] T_{i}^{(3)}= \\
\left(T_{i}^{(3)}\right)^{t}\left(\mathcal{R}_{i}^{(2)}\right)^{-1} T_{i}^{(3)}-\left(T_{i}^{(3)}\right)^{t}\left(\mathcal{R}_{i}^{(2)}\right)^{-1} U_{i}^{(2)}\left(G_{i}^{(2)}\right)^{-1}\left(V_{i}^{(2)}\right)^{t}\left(\mathcal{R}_{i}^{(2)}\right)^{-1} T_{i}^{(3)}
\end{gathered}
$$

It holds

$$
\begin{gathered}
\left(T_{i}^{(3)}\right)^{t}\left(\mathcal{R}_{i}^{(2)}\right)^{-1} T_{i}^{(3)}=T_{i 0}^{t} T_{i 0}, \quad\left(T_{i}^{(3)}\right)^{t}\left(\mathcal{R}_{i}^{(2)}\right)^{-1} U_{i}^{(2)}=\left(T_{i 0}^{t} A_{i 0}, T_{i 0}^{t}\right), \\
\left(V_{i}^{(2)}\right)^{t}\left(\mathcal{R}_{i}^{(2)}\right)^{-1} T_{i}^{(3)}=\left(\begin{array}{c}
A_{i 0}^{t} T_{i 0} \\
\left.-T_{i 0}\right)
\end{array}\right) .
\end{gathered}
$$

Therefore

$$
\left(T_{i}^{(3)}\right)^{t}\left(R_{i}^{(2)}\right)^{-1} T_{i}^{(3)}=T_{i 0}^{t} T_{i 0}-\left(T_{i 0}^{t} A_{i 0}, T_{i 0}^{t}\right)\left(\begin{array}{cc}
\hat{G}_{i}^{(2)} & A_{i 0}^{t} \\
-A_{i 0} & 0
\end{array}\right)^{-1}\left(\begin{array}{c}
A_{i 0}^{t} T_{i 0} \\
-T_{i 0}
\end{array}\right)
$$

Now let

$$
\left(\begin{array}{cc}
K & L \\
M & N
\end{array}\right)=\left(\begin{array}{cc}
\hat{G}_{i}^{(2)} & A_{i 0}^{t} \\
-A_{i 0} & 0
\end{array}\right)^{-1}
$$

According to $[6]$

$$
\begin{aligned}
N & =\left[A_{i 0}\left(\hat{G}_{i}^{(2)}\right)^{-1} A_{i 0}^{t}\right]^{-1} \\
L & =-\left(\hat{G}_{i}^{(2)}\right)^{-1} A_{i 0}^{t}\left[A_{i 0}\left(\hat{G}_{i}^{(2)}\right)^{-1} A_{i 0}^{t}\right]^{-1} \\
M & =\left[A_{i 0}\left(\hat{G}_{i}^{(2)}\right)^{-1} A_{i 0}^{t}\right]^{-1} A_{i 0}\left(\hat{G}_{i}^{(2)}\right)^{-1} \\
K & =\left(\hat{G}_{i}^{(2)}\right)^{-1}-\left(\hat{G}_{i}^{(2)}\right)^{-1} A_{i 0}^{t}\left[A_{i 0}\left(\hat{G}_{i}^{(2)}\right)^{-1} A_{i 0}^{t}\right]^{-1} A_{i 0}\left(\hat{G}_{i}^{(2)}\right)^{-1} .
\end{aligned}
$$

Now, if we use (42)-(45) in (40) we obtain

$$
\left(T_{i}^{(3)}\right)^{t}\left(R_{i}^{(2)}\right)^{(-1)} T_{i}^{(3)}=T_{i 0}^{t}\left[A_{i 0}\left(\hat{G}_{i}^{(2)}\right)^{-1} A_{i 0}^{t}\right]^{-1} T_{i 0}-T_{i 0}^{t} T_{i 0} .
$$

Thus,

$$
\hat{G}^{(3)}=D_{0}^{-1}+A_{0}^{t} A_{0}+\sum_{i=1}^{2} T_{i 0}^{t}\left(\hat{T}_{i 0}-T_{i 0}\right) .
$$

We remember that the Cholesky decomposition of matrix $A_{i 0}\left(\hat{G}_{i}^{(2)}\right)^{-1} A_{i 0}^{t}$ has been performed during the procedure Findy applied on matrix $R_{i}^{(2)}, i=1,2$. Thus, this decomposition is available already, only triangular solver is used for the computation of $\hat{T}_{i 0}, i=1,2$ in this step. Having the values of $\hat{G}^{(3)}$ and $\left(\hat{v}_{1}^{(3)}, \hat{v}_{2}^{(3)}\right)^{t}$ we can solve the system

$$
\left(\begin{array}{cc}
\hat{G}^{(3)} & A_{0}^{t} \\
-A_{0} & 0
\end{array}\right)\left(\begin{array}{l}
q_{1}^{(3)} \\
q_{2}^{(3)}
\end{array}\right)=\left(\begin{array}{l}
\hat{v}_{1}^{(3)} \\
\hat{v}_{2}^{(3)}
\end{array}\right)
$$


The standard elimination process applied on this system yields

$$
\begin{aligned}
{\left[\left(A_{0}\left(\hat{G}^{(3)}\right)^{-1} A_{0}^{t}\right] q_{2}^{(3)}\right.} & =A_{0}\left(\hat{G}^{(3)}\right)^{-1} \hat{v}_{1}^{(3)}+\hat{v}_{2}^{(3)} \\
\hat{G}^{(3)} q_{1}^{(3)} & =\hat{v}_{1}^{(3)}-A_{0}^{t} q_{2}^{(3)} .
\end{aligned}
$$

Thus, to solve (48) the following procedure is required:

$\operatorname{PROCEDURE} \operatorname{Updy}\left(\hat{G}^{(3)}, A_{0}, \hat{v}_{1}^{(3)}, \hat{v}_{2}^{(3)}\right)$

(a) Form the Cholesky decomposition of $\hat{G}^{(3)}$

(b) Solve the systems $\hat{G}^{(3)} B_{0}=A_{0}^{t}$

(c) Form the Cholesky decomposition of $A_{0} B_{0}$

(d) Solve the systems (49) and (50).

\subsection{Solving the Equation $\mathcal{R}^{(3)} s^{(3)}=U^{(3)} q^{(3)}$}

The system $\mathcal{R}^{(3)} s^{(3)}=U^{(3)} q^{(3)}$ could be solved in a similar way as in Sect. 3.1. The right-hand side equals

$$
U^{(3)} q^{(3)}=\left(\begin{array}{cc}
A_{0} & I_{m_{0}} \\
T_{1}^{(3)} & 0 \\
T_{2}^{(3)} & 0
\end{array}\right)\left(\begin{array}{c}
q_{1}^{(3)} \\
q_{2}^{(3)}
\end{array}\right)=\left(\begin{array}{c}
A_{0} q_{1}^{(3)}+q_{2}^{(3)} \\
T_{1}^{(3)} q_{1}^{(3)} \\
T_{2}^{(3)} q_{1}^{(3)}
\end{array}\right) .
$$

Thus, the system has the form

$$
\mathcal{R}^{(3)} s^{(3)}=\left(\begin{array}{ccc}
I_{m_{0}} & & \\
& R_{1}^{(2)} & \\
& & R_{2}^{(2)}
\end{array}\right)\left(\begin{array}{c}
s_{0}^{(3)} \\
s_{1}^{(3)} \\
s_{2}^{(3)}
\end{array}\right)=\left(\begin{array}{ccc}
A_{0} q_{1}^{(3)}+q_{2}^{(3)} \\
T_{1}^{(3)} & q_{1}^{(3)} \\
& T_{2}^{(3)} & q_{1}^{(3)}
\end{array}\right),
$$

from which we obtain independent equations

$$
\begin{aligned}
s_{0}^{(3)} & =A_{0} q_{1}^{(3)}+q_{2}^{(3)} \\
R_{i}^{(2)} s_{i}^{(3)} & =T_{i}^{(3)} q_{1}^{(3)}, i=1,2 .
\end{aligned}
$$

The last two equations are again solvable by the procedure Findy as in Sect. 3.1 with the right-hand side $T_{i}^{(3)} q_{1}^{(3)}, i=1,2$. But, owing to the structure of this vector, where only the first $m_{i 0^{-}}$entries are nonzero, we suggest for its computation a modification the already mentioned procedure Findy.

The solution $s_{i}^{(3)}$ of (54) can be expressed as $s_{i}^{(3)}=\hat{p}_{i}^{(3)}-\hat{s}_{i}^{(3)}$, where $\hat{p}_{i}^{(3)}$ and $\hat{s}_{i}^{(3)}, i=1,2$ fulfil

$$
\begin{aligned}
& \mathcal{R}_{i}^{(2)} \hat{p}_{i}^{(3)}=T_{i}^{(3)} q_{1}^{(3)} \\
& G_{i}^{(2)} \hat{q}_{i}^{(3)}=\left(V_{i}^{(2)}\right)^{t} \hat{p}_{i}^{(3)} \\
& \mathcal{R}_{i}^{(2)} \hat{s}_{i}^{(3)}=U_{i}^{(2)} \hat{q}_{i}^{(3)} .
\end{aligned}
$$


In the matrix form

$$
\mathcal{R}_{i}^{(2)} \hat{p}_{i}^{(3)}=\left(\begin{array}{cccc}
I_{m_{i 0}} & & & \\
& R_{i 1} & & \\
& & R_{i 2} & \\
& & & R_{i 3}
\end{array}\right)\left(\begin{array}{c}
\hat{p}_{i 0}^{(3)} \\
\hat{p}_{i 1}^{(3)} \\
\hat{p}_{i 2}^{(3)} \\
\hat{p}_{i 3}^{(3)}
\end{array}\right)=\left(\begin{array}{c}
T_{i 0} q_{1}^{(3)} \\
0 \\
0 \\
0
\end{array}\right),
$$

from which we have immediately

$$
\begin{aligned}
& \hat{p}_{i 0}^{(3)}=T_{i 0} q_{1}^{(3)}, \quad i=1,2 \\
& \hat{p}_{i j}^{(3)}=0, \quad i=1,2 \quad j=1,2,3 .
\end{aligned}
$$

To find the solution of (56) means to solve the following matrix equation

$$
\left(\begin{array}{cc}
\hat{G}_{i}^{(2)} & A_{i 0}^{t} \\
-A_{i 0} & 0
\end{array}\right)\left(\begin{array}{c}
\hat{q}_{i 1}^{(3)} \\
\hat{q}_{i 2}^{(3)}
\end{array}\right)=\left(\begin{array}{c}
A_{i 0}^{t} \hat{p}_{i 0}^{(3)} \\
-\hat{p}_{i 0}^{(3)}
\end{array}\right) .
$$

The last equation (57) represents the system

$$
\mathcal{R}_{i}^{(2)} \hat{s}_{i}^{(3)}=\left(\begin{array}{cccc}
I_{m_{i 0}} & & & \\
& R_{i 1} & & \\
& & R_{i 2} & \\
& & & R_{i 3}
\end{array}\right)\left(\begin{array}{c}
\hat{s}_{i 0}^{(3)} \\
\hat{s}_{i 1}^{(3)} \\
\hat{s}_{i 2}^{(3)} \\
\hat{s}_{i 3}^{(3)}
\end{array}\right)=\left(\begin{array}{c}
A_{i 0} \hat{q}_{i 1}^{(3)}+\hat{q}_{i 2}^{(3)} \\
T_{i 1} \hat{q}_{i 1}^{(3)} \\
T_{i 2} \hat{q}_{i 1}^{(3)} \\
T_{i 3} \hat{q}_{i 1}^{(3)}
\end{array}\right),
$$

from which we have

$$
\begin{aligned}
\hat{s}_{i 0}^{(3)} & =A_{i 0} \hat{q}_{i 1}^{(3)}+\hat{q}_{i 2}^{(3)}, \quad i=1,2 \\
R_{i j} \hat{s}_{i j}^{(3)} & =T_{i j} \hat{q}_{i 1}^{(3)}, \quad i=1,2 \quad j=1,2,3 .
\end{aligned}
$$

With the vector $\hat{s}_{i j}^{(3)}$ available, we have the result

$$
s_{i}^{(3)}=\hat{p}_{i}^{(3)}-\hat{s}_{i}^{(3)}=\left(\begin{array}{c}
\hat{p}_{i 0}^{(3)}-\hat{s}_{i 0}^{(3)} \\
-\hat{s}_{i 1}^{(3)} \\
-\hat{s}_{i 2}^{(3)} \\
-\hat{s}_{i 3}^{(3)}
\end{array}\right), \quad i=1,2 .
$$

Finally, the computing of $s_{i}^{(3)}, i=1,2$ consist of the following steps:

PROCEDURE Findysparse $\left(\mathcal{R}_{i}^{(2)}, A_{i 0}, T_{i 1}, T_{i 2}, T_{i 3}, T_{i}^{(3)} q_{1}^{(3)}, s_{i}^{(3)}\right)$

1. Set $\hat{p}_{i 0}^{(3)}=T_{i 0} q_{1}^{(3)}, \quad i=1,2$

2. Solve the system $(61)$

3. (a) Set $\hat{s}_{i 0}^{(3)}=A_{i 0} \hat{q}_{i 1}^{(3)}+\hat{q}_{i 2}^{(3)}, \quad i=1,2$

(b) Solve $R_{i j} \hat{s}_{i j}^{(3)}=T_{i j} \hat{q}_{i 1}^{(3)}, \quad i=1,2 ; j=1,2,3$

4. Set $s_{i}^{(3)}=\hat{p}_{i}^{(3)}-\hat{s}_{i}^{(3)} \quad i=1,2$. 
Note that the Cholesky decomposition of the system matrices is available in step 2 and $3(b)$. These decomposition has been computed by the procedure Findy. In the end, the result of a three-stage stochastic model problem equals $d y^{(3)}=p^{(3)}-s^{(3)}$. This difference is obtained by the following computational process :

1. Call Findy $\left(\mathcal{R}_{i}^{(2)}, A_{i 0}, D_{i 0}, T_{i 1}, \ldots, T_{i 3}, b_{i}^{(3)}, p_{i}^{(3)}\right), \quad i=1,2$,

2. Call $\operatorname{Updy}\left(\hat{G}^{(3)}, A_{0}, \hat{v}_{1}^{(3)}, \hat{v}_{2}^{(3)}\right)$

3. Call Findysparse $\left(\mathcal{R}_{i}^{(2)}, A_{i 0}, T_{i 1}, T_{i 2}, T_{i 3}, T_{i}^{(3)} q_{1}^{(3)}, s_{i}^{(3)}\right) \quad \mathrm{i}=1,2$

4. Form $d y^{(3)}$ as difference $p^{(3)}-s^{(3)}$.

The procedures in steps 1 and 3 are independent and can be computed at the same time. Step 2 represents a binding of existing two-stage models and enables to calculate $s_{0}^{(3)}$ and the parameter $q_{i}^{(3)}, \quad \mathrm{i}=1,2$ for Findysparse(). Roughly, the process may be symbolically written as:

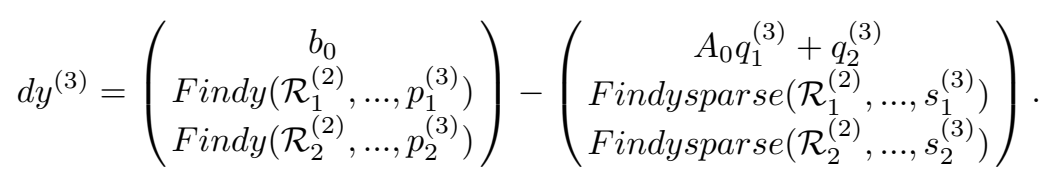

\section{Conclusion}

The aim of our paper has been to use the BQ factorization technique for threestage stochastic program in a framework of an interior point method. As we can see, this technique leads to the solution of independent subproblems. Moreover, these subproblems are again scalable into smaller linear system of equations. The whole process contains a serial coordination step, but the range of a sequential computation is not critical for large-scale stochastic program. A parallel implementation of this algorithm within an interior point code and an extension to the multistage model will be topics of our future work.

\section{References}

1. Vladimirou, H. Zenios, S.A.: Scalable parallel computations for large-scale stochastic programming,Annals of Oper. Res. 90,1999, pp.87-129.

2. Birge, J.R., Holmes, D.F.: Efficient solution of two-stage stochastic linear programs using interior point methods, Comput. Optim. Appl., 1, 1992,pp. 245-276.

3. Birge, J.R., Qi, L.: Computing block-angular Karmarkar projections with applications to stachastic programming, Management Sci., 34,1988, pp.1472-1479.

4. Jessup, E.R., Yang, D., Zenios, S.A.: Parallel factorization of structured matrices arising in stochastic programming, SIAM J.Optimization, Vol. 4., No.4,1994, pp. 833-846.

5. Golub,G.H., Van Loan, Ch.F.: Matrix Computations, The Johns Hopkins University Press, 1996.

6. A.Ralston : A first course in numerical analysis, McGraw-Hill, inc., New York, 1965. 